\title{
Numerical modelling of destruction of a drop of non-Newtonian fluid in a gas flow
}

\author{
Anna Shebeleva ${ }^{1,2^{*}}$, Andrey Minakov ${ }^{1,2}$, Alexander Lobasov ${ }^{1}$, and Alexander Shebelev ${ }^{1,2}$ \\ ${ }^{1}$ Siberian Federal University, Krasnoyarsk, Russia \\ ${ }^{2}$ Kutateladze Institute of Thermophysics, Russian Academy of Sciences, Novosibirsk, Russia
}

\begin{abstract}
The research presents the numerical modeling findings of the secondary breakup of droplets of coal water slurries containing petrochemicals (CWSP) droplet with the filter cake content of $50 \%$ for different Weber number values. The modeling method of the secondary droplet breakup is based on the VOF method for interface resolution, LES model for describing turbulence, and the technology of adapted dynamic grids. This technology enables the grid to be automatically concentrated in the region of large solution gradients during the calculation. The implementation of such a highly detailed grid allowed resolving secondary droplets with the dimensions down to $15 \mu \mathrm{m}$. We established the droplet breakup modes depending on the Weber number ranging from 36 to 342 . The structure of the stream behind droplets was studied in detail and the numerical method was tested. The results are in good agreement with the results of known experiments.
\end{abstract}

\section{Introduction}

Dispersed systems comprised of water, milled coal or combustible coal processing wastes, are quite a promising alternative fuel for energy generation. Such fuels are referred to as coal water slurries containing petrochemicals or composite liquid fuels. The main method of slurry fuel combustion is its preliminary atomization in a combustion chamber. This appreciably expands the contact area of the fuel with the oxidizer and intensifies heat and mass exchange. The jet atomization is accompanied by its primary breakup and formation of liquid droplets that suffer deformation under aerodynamic force, which can lead to droplet breakup (secondary breakup). The primary task in the development of new combustion technologies is to establish the dependence of this process on fuel properties and conditions of fuel supply into the chamber. Therefore, the aim of this study is to test the numerical method of destruction of individual drops of non-Newtonian fluids in the flow.

\section{Simulation model}

Nowadays, the gas-droplet flows can be described using a wide variety of mathematical models. They are distinguished by methods of gas and particle movement description,

* Corresponding author: an riv@mail.ru 
accepted assumptions and limitations of use. As a rule, a suitable model is chosen with due consideration of available information about the flow structure and the required description accuracy. The methods of solving problems with moving interfaces are reviewed most comprehensively in [1]. Nowadays, the high efficacy and implementation simplicity of the volume-of-fluid method (VOF) [2] make it the most popular one among the algorithms of continuous volume markers. It has proven itself in the calculation of flows with a free surface $[1,3]$. The idea of the method is that a fluid and a gas are regarded as a single twocomponent medium; the spatial distribution of phases within the calculation domain is determined by the special marker function $\mathrm{F}(\mathrm{x}, \mathrm{y}, \mathrm{z}, \mathrm{t})$. The volumetric fraction of the liquid phase in the calculation cell is as follows: $F(x, y, z, t)=0$ for an empty cell, $F(x, y, z, t)=1$ for a cell entirely filled with liquid, $0<\mathrm{F}(\mathrm{x}, \mathrm{y}, \mathrm{z}, \mathrm{t})<1$ for a cell with the interface passing through it.

Since the free surface moves along with the liquid, the free boundary movement in the space is tracked by solving the transfer equation of liquid phase volume fraction in the cell:

$$
\frac{\partial F}{\partial t}+\mathbf{V} \cdot \nabla F=0
$$

where $\mathbf{V}$ is the velocity vector of the two-phase medium found from solving a simultaneous hydrodynamics equation consisting of a mass conservation equation and a continuity equation:

$$
\frac{d \rho}{d t}+\nabla(\rho \cdot \mathbf{V})=0
$$

and a motion equation or the law of momentum conservation:

$$
\frac{d \rho \mathbf{V}}{d t}+\nabla(\rho \mathbf{V} \times \mathbf{V})=-\nabla p+\nabla(\boldsymbol{\tau})+\mathbf{F}
$$

where $\boldsymbol{\tau}$ is the tensor of viscous stresses, $\mathbf{F}$ is the vector of volume forces, $\mathrm{p}$ is static pressure, $\rho$ is the density of a two-phase medium.

The components of the tensor of viscous stresses $\boldsymbol{\tau}_{\mathrm{ij}}$, where $\mu$ is the dynamic viscosity of a two-phase medium, $\mathrm{U}_{\mathrm{ij}}$ are the components of the velocity vector.

$$
\boldsymbol{\tau}_{i j}=\mu\left(\frac{d U_{i}}{d x_{j}}+\frac{d U_{j}}{d x_{i}}-\frac{2}{3} \delta_{i j} \frac{d U_{k}}{d x_{k}}\right)
$$

The density and molecular viscosity of the two-component medium under study are determined through the volumetric fraction of the liquid in a cell using the mixture rule:

$$
\begin{aligned}
& \rho=\rho_{1} F+(1-F) \rho_{2} \\
& \mu=\mu_{1} F+(1-F) \mu_{2}
\end{aligned}
$$

Since CWSs can exhibit non-Newtonian properties in certain cases, the non-Newtonian flows were modeled using the approach from [4], where a medium is considered a nonlinear viscous liquid with the introduction of effective viscosity, which normally depends on the shear rate. The shear rate is, in general terms, the second invariant of the tensor of deformation rates: 


$$
\dot{\gamma}=\sqrt{0.5 \cdot \mathbf{D} \cdot \mathbf{D}}
$$

The components of the tensor of deformation rates $\mathbf{D}$ are as follows:

$$
\mathbf{D}_{i j}=\frac{\partial U_{i}}{\partial x_{j}}+\frac{\partial U_{j}}{\partial x_{i}}
$$

Thus, within the given model, a CWSP can be both a viscous Newtonian liquid and a non-Newtonian viscoplastic liquid whose behavior can be described by rheological model Bingham-plastic (Bingham liquid):

$$
\mu_{1}(\dot{\gamma})=\frac{k \dot{\gamma}+\tau_{0}}{\dot{\gamma}}
$$

where $\mathrm{k}$ are coefficient of rheological model, $\boldsymbol{\tau}_{\mathbf{0}}$ is the yield point of a viscoplastic liquid.

The consideration of liquid flows with an interface draws special attention to the surface tension. Most frequently, the surface tension forces within the VOF method are modeled by CSF algorithm which implies the introduction of the additional volume force. This algorithm is described in detail in the work [3]. Another important factor in the droplet breakup calculation is turbulence modeling. The present work uses LES model [5] to model the turbulence. This model requires the solution of filtered Navier-Stokes equations to describe turbulent flows. The method of solving equations and the main peculiarities of numerical studies are described elsewhere $[1,3]$.

\section{Results and discussion}

The above described method was used to model secondary breakup of a CWSP droplet with the filter cake content of $50 \%$ for different Weber number values. The physical properties of a CWSP were specified according to the data from [6]. The parameters of cases covered in the calculation are presented in Table 1. The secondary breakup of droplets of homogeneous liquids occurs under the aerodynamic force exceeding the surface tension forces. Quantitatively, the correlation of these forces is determined by the value of the deformation criterion the Weber number.

Table 1. The parameters of cases.

\begin{tabular}{|c|c|c|c|c|c|c|c|}
\hline Case & $\mathrm{D} 0[\mathrm{~mm}]$ & $\rho[\mathrm{kg} / \mathrm{m} 3]$ & $\tau 0[\mathrm{~Pa}]$ & $\mathrm{k}[\mathrm{Pa} * \mathrm{~s}]$ & $\sigma[\mathrm{N} / \mathrm{m}]$ & $\mathrm{V}[\mathrm{m} / \mathrm{s}]$ & $\mathrm{We}$ \\
\hline 1 & 4.6 & 1179 & 1.96 & 0.06 & 0.0613 & 19.7 & 36 \\
\hline 2 & 4.8 & 1111 & 2.12 & 0.14 & 0.066 & 23.2 & 48 \\
\hline 3 & 4.8 & 1111 & 2.12 & 0.14 & 0.066 & 61.9 & 342 \\
\hline
\end{tabular}

The calculation and the experimental data [6] on the dynamics of a CWS droplet breakup for the considered breakup modes are compared in Figs. 1-3.

The first breakup mode ( $\mathrm{We}=36$ and $\mathrm{We}=48$ ) is characterized at the initial breakup stage by the behavior similar to the parachute breakup mode. The droplet swells into a shell. However, the flow then tears off a thin film from it, and the droplet turns into a ring, which further breaks up into separate fragments. Other researchers refer to such mode as a hole breakup. We can note that at $\mathrm{We}=36$ after the disruption of the liquid shell, a ring is 
formed, while in the center a relatively large fragment of the droplet remains with the dimensions of up to $20 \%$ of the initial droplet size. This fragment then decays rapidly.

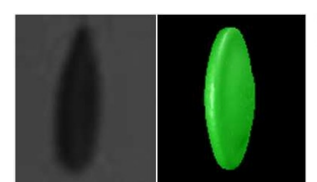

$15.3 \mathrm{~ms}$

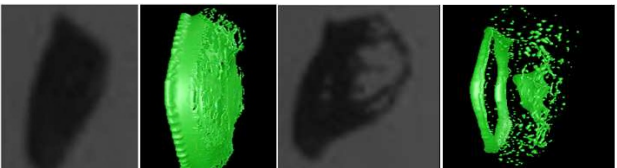

$16.3 \mathrm{~ms}$
$17.3 \mathrm{~ms}$

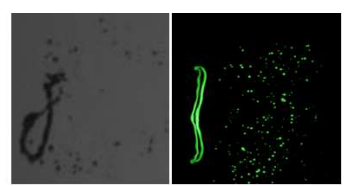

$22.3 \mathrm{~ms}$

Fig. 1. Comparison of experimental [6] (left-hand side) and calculated shape of CWS droplet surface at different moments of time at the $\mathrm{We}=36$.

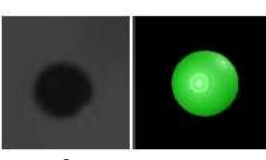

$0 \mathrm{~ms}$

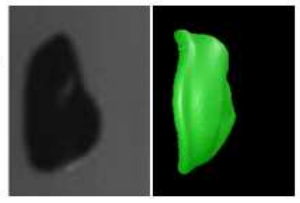

$10.7 \mathrm{~ms}$

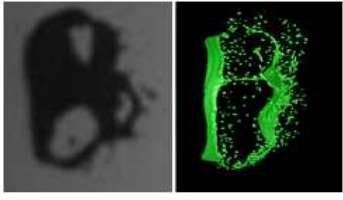

$12.7 \mathrm{~ms}$

Fig. 2. Comparison of experimental [6] (left-hand side) and calculated shape of CWS droplet surface at different moments of time at the $\mathrm{We}=48$.

The second mode $(\mathrm{We}=342)$ actually represents the catastrophic droplet breakup. In this mode, the droplet rapidly turns into a disc; however, unlike in the previous case, this disc does not swell in the direction of the flow, and the gas flow continuously blows off droplets from its external edge. As a result, the droplet very quickly turns into spray.

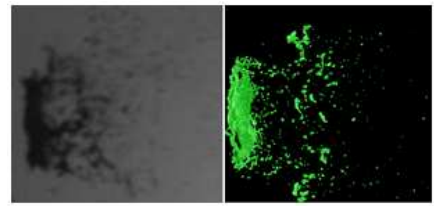

$3.7 \mathrm{~ms}$

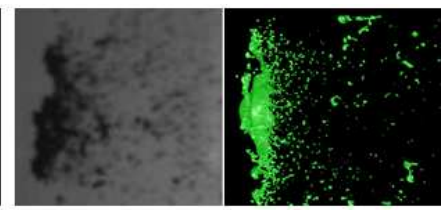

$4.7 \mathrm{~ms}$

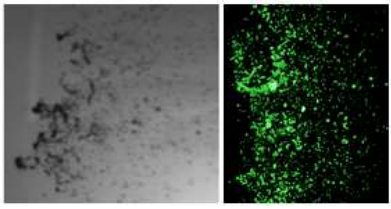

$7 \mathrm{~ms}$

Fig. 3. Comparison of experimental [6] (left-hand side) and calculated shape of CWS droplet surface at different moments of time at the $\mathrm{We}=342$.

The analysis of the modeling results shows that for both of the modes under study the calculation satisfactorily describes the experimental data in terms of both the breakup process itself and the shape of droplet surface and their separate fragments at different moments of time. This confirms the operability of the numerical technique and the possibility of its application for predicting the behavior of droplets non-Newtonian fluid.

The reported study was funded by RFBR according to the research project № 18-38-00565, and Ministry of Education and Science of the Russian Federation Government contract with Siberian Federal University in 2018 (No. 16.8368.2017).

\section{References}

1. S. Osher, J.A. Sethian, Journal of computational physics 79 (1988)

2. C.W. Hirt, B.D. Nichols, Journal of computational physics 39 (1981)

3. A.A. Shebeleva, A.V. Minakov, et.al., MATEC Web of Conferences (2015)

4. A.A. Gavrilov., A.A. Dekterev, Computation technologies 17 (2012)

5. J. Smagorinsky. J. Monthly Weather Review 91 (1963) 
6. Zhao Hui, Liu Hai-Feng, Xu Jian-Liang, Li Wei-Feng, Phys. Fluids. 23 (2011) 